\title{
Prenatal thyroid hormones accelerate postnatal growth and telomere shortening in wild great tits
}

Bin-Yan Hsu ${ }^{1}$, Nina Cossin-Sevrin ${ }^{1,2}$, Antoine Stier $^{1,3^{*}}$, Suvi Ruuskanen ${ }^{1,4} \$$

${ }^{1}$ Department of Biology, University of Turku, Finland

${ }^{2}$ Université de Strasbourg, Centre National de la Recherche Scientifique, Institut

Pluridisciplinaire Hubert Curien, UMR 7178, 67087 Strasbourg, France

3 Univ Lyon, Université Claude Bernard Lyon 1, CNRS, ENTPE, UMR 5023 LEHNA, F-69622,

1 Villeurbanne, France

${ }^{4}$ Department of Biological and Environmental Science, University of Jyväskylä

*joint senior authors

\$Corresponding author: suvi.k.ruuskanen@gmail.com

Running headline: Prenatal thyroid hormones shorten telomeres 


\section{Abstract}

Early-life environment is known to affect later-life health and disease, which could be mediated by the early-life programming of telomere length, a key hallmark of ageing. According to the fetal programming of telomere biology hypothesis, variation in prenatal exposure to hormones is likely to influence telomere length. Yet the contribution of key metabolic hormones, i.e. thyroid hormones (THs), has been largely ignored. We recently showed that in contrast to predictions, exposure to elevated prenatal THs increased postnatal telomere length in wild collared flycatchers, but the generality of such effect, its underlying proximate mechanisms and consequences on survival have not been investigated. We therefore conducted a comprehensive study evaluating the impact of THs on potential drivers of telomere dynamics (growth, post-natal THs, mitochondria and oxidative stress), telomere length and medium-term survival using wild great tits as a model system. While prenatal THs did not significantly affect telomere length after hatching (i.e. day 7), they influenced postnatal telomere shortening (i.e. shorter telomeres at day 14 and the following winter) but not apparent survival. Circulating THs, mitochondrial density or oxidative stress biomarkers were not significantly influenced, whereas TH-supplemented group showed accelerated growth, which may explain the observed delayed effect on telomeres. We discuss several alternative hypotheses that may explain the contrast with our previous findings in flycatchers. Given that shorter telomeres in early life tend to be carried until adulthood and are often associated with decreased survival prospects, the effects of prenatal THs on telomeres may have long-lasting effects on senescence.

Keywords: developmental programming, thyroid hormone, telomere, ageing, mitochondria, metabolism, oxidative stress, maternal effects 


\section{Introduction}

Early-life environment has been repeatedly observed to affect adult health and survival prospects in human and non-human vertebrates (Barnes and Ozanne, 2011; Godfrey and Barker, 2001; Metcalfe and Monaghan, 2001). While the mechanisms underlying such delayed effects remained somewhat elusive (Barnes and Ozanne, 2011), the early-life programming of telomere length (i.e. the protective end caps of chromosomes) has emerged as a key candidate (Entringer et al., 2018). Telomere length is considered as a hallmark of ageing (López-Otín et al., 2013) since telomeres shorten with age, and shorter telomeres are often predictive of lower survival or lifespan in both epidemiological and experimental studies (Arbeev et al., 2020; Heidinger et al., 2012; Muñoz-Lorente et al., 2019; Wilbourn et al., 2018). The prenatal hormonal environment, such as exposure to elevated glucocorticoid levels, has been coined as an important factor influencing early-life telomere length and its associated long-term outcomes (Criscuolo et al., 2020; Haussmann et al., 2012; Marchetto et al., 2016; Parolini et al., 2019). While there has been a considerable interest in prenatal glucocorticoids (Haussmann et al. 2012; Tissier, Williams, and Criscuolo 2014; Noguera, da Silva, and Velando 2020) and to a lesser extent androgens (Parolini et al., 2019; Tissier et al., 2014) in the context of the 'fetal programming of telomere biology' hypothesis (Entringer et al., 2018), the potential impact of prenatal thyroid hormones has been mostly ignored so far (Stier et al. 2020).

Thyroid hormones (THs) are key coordinators of development and metabolism (McNabb, 2007), which are transferred from mothers to offspring (Ruuskanen and Hsu, 2018). Variation in exposure to prenatal thyroid hormones (T3, triiodothyronine, and $\mathrm{T} 4$, thyroxine) could influence telomere length via several mutually non-exclusive proximate pathways: (i) Prenatal THs can stimulate growth (yet results are inconsistent across studies and species, 
(Hsu et al., 2020, 2019a, 2017; Medici et al., 2013; Ruuskanen et al., 2016a; Sarraude et al., 2020a; Sarraude et al., 2020b; Vrijkotte et al., 2017; Zhang et al., 2019), which can directly contribute to telomere attrition through increasing cellular division (Monaghan and Ozanne, 2018; Stier et al., 2020), or indirectly accelerate telomere shortening through increasing oxidative stress (Monaghan and Ozanne, 2018; Reichert and Stier, 2017; Smith et al., 2016). (ii) Elevated TH levels are often associated with higher metabolic rates (Liu et al., 2006; Mullur et al., 2014; Welcker et al., 2013) and stimulate mitochondrial aerobic metabolism (Cioffi et al., 2013), both of which can potentially increase reactive oxygen species (ROS) and oxidative damage (Stier, Massemin, and Criscuolo 2014), accelerating telomere shortening (Reichert and Stier, 2017). It was recently shown that exposure to elevated prenatal TH levels can lead to a sex-specific increase in metabolic rate and circulating thyroid hormone levels shortly after hatching (rock pigeons Columba livia, Hsu et al. 2017, but see Ruuskanen et al. 2016), which suggests that prenatal hormones may program postnatal metabolism and $\mathrm{TH}$-axis function. (iii) The 'metabolic telomere attrition hypothesis' (Casagrande and Hau, 2019) postulates that telomere shortening might be adaptive and have signaling functions related to metabolic demand (i.e. accentuated shortening when catabolism is favored over anabolism via mTOR inhibition). Since THs can have both anabolic and catabolic actions (Mullur et al., 2014), predictions can be made in both directions between prenatal THs and telomere length. From an evolutionary perspective, increased offspring metabolism or growth, which may be beneficial for the mother/offspring, diverts resources from somatic maintenance if resources are limited. This can accelerate damage to biomolecules and/or decrease repair/maintenance processes, and therefore accentuate telomere shortening. Therefore, prenatal TH levels would be expected to vary in relation to predicted environmental conditions (as observed in 
terms of temperature and laying order, e.g. Ruuskanen, Groothuis, et al. 2016; Hsu, Verhagen, et al. 2019) to optimize this trade-off.

In contrast to most predictions relating prenatal THs to telomere length (see above), we recently reported that prenatal exposure to experimentally elevated THs increased telomere length in nestlings of a wild passerine, the collared flycatcher (Ficedula albicollis, Stier et al. 2020). To better understand the potential generality of this surprising finding as well as assess underlying mechanisms and potential carry-over effects of variation in prenatal THs on later life-stages and survival, we conducted a more detailed study in another passerine species, the great tit (Parus major). The aim of this study was to comprehensively investigate the influence of prenatal THs on growth, oxidative stress, plasma THs, mitochondrial density and telomere dynamics as well as survival via an experimental manipulation of prenatal THs in a wild population. We monitored offspring multiple times during development and as juveniles a few months after fledging. Based on the majority of prior literature, we would predict that elevated prenatal THs could lead to faster growth, increased plasma THs, oxidative stress and mitochondrial density, ultimately accelerating telomere shortening. Alternatively, if our previous finding in the collared flycatcher reflected a general pattern, we would predict that despite accelerating growth, elevated prenatal THs could increase telomere length. We also predict that elevated prenatal TH could increase post-fledging survival of the juveniles (e.g. both due to accelerated postnatal growth and potential beneficial effects for thermoregulation under low autumn-winter temperatures). However, longer-term survival, that we were not able to evaluate here, could be decreased for example due to shorter telomeres. As a previous study in the same species reported sex-dependent effects of prenatal THs on growth (Ruuskanen et al., 2016), we may also expect the effects on physiology and survival to differ between sexes. 


\section{Methods}

The experiment and all methods we used were in accordance with all relevant guidelines and regulations and have been approved by the Animal Experiment Board of the Administrative Agency of South Finland (ESAVI/2902/2018) and the Environmental Center of Southwestern Finland (license number VARELY549/2018). The experiment was conducted in 2018 in a nest box population (314 nest-boxes distributed over seven forest plots) on the island of Ruissalo in southwestern Finland $\left(60^{\circ} 25^{\prime} \mathrm{N}, 22^{\circ} 10^{\prime} \mathrm{E}\right)$.

\section{Field experiment}

The nest boxes were monitored with five-day intervals to track egg laying. Yolk T3 and T4 (i.e. a combined injection of the two hormones) levels were elevated in half of the nests (n $=21 \mathrm{TH}$ nests and $\mathrm{n}=21$ control nests) by injection into the egg, following methods in Ruuskanen, Darras, et al. (2016). Control nests were injected with the vehicle $(0.9 \% \mathrm{NaCl})$ only. The TH concentration in great tit eggs is in the population is $0.11 \pm 0.04 \mathrm{ng} /$ yolk for T3 and $0.96 \pm 0.29 \mathrm{ng} /$ yolk for T4 (Ruuskanen et al., 2019). We aimed to raise the amount of yolk TH by $2 \mathrm{SD}$ via injection into the egg yolk, a dose that has been recommended in relation to the natural hormone range of the study species (Podmokla et al., 2018). This corresponded to target doses of $0.041 \mathrm{ng} /$ yolk for T3 and $0.325 \mathrm{ng} /$ yolk for T4. To make sure injections would be performed on unincubated eggs mimicking maternal hormone levels (great tits can start incubation before the clutch is complete), injections were conducted on the day the $5^{\text {th }}$ egg was laid to all eggs in the clutch. Thereafter, injections were conducted each day for the newly laid egg. 
day 2 (mass only), 7 and 14 post-hatching. All nestling measurements were conducted

half of the brood were cross-fostered with same-age nestlings in another nest (preferentially and control-injected eggs experiencing the same postnatal environment. On day 7 a blood sample (ca $40 \mu \mathrm{l}$ ) was taken, kept on ice, centrifuged within 8 hours, and RBCs frozen at $-80 \circ \mathrm{C}$ data from the nests which were not temperature-manipulated for day 14 and in juveniles ( $N$ = 19 nests, 99 nestlings on day 14). Yet, for day 2 and day 7 measurements (prior to the temperature manipulation), we prefer to include all data ( $N=41$ nests, 218 nestlings on $d 2$ and 221 nestlings on $\mathrm{d7}$ ) to make use of the larger sample size.

To study long-term effects of prenatal thyroid hormones and offspring apparent postfledging survival, we recaptured great tits during the following autumn-winter using mist- 
forest plot. In addition, 5 birds were captured at a constant effort site $>3 \mathrm{~km}$ from the study plots, suggesting that all stations were potentially accessible to all birds. Circa $20 \mathrm{~kg}$ of peeled sunflower seeds and $2 \mathrm{~kg}$ of fat were provided at each station, checked and filled bi-weekly, and consumption noted. Only in a very few cases the feeders were completely empty. Mistnetting was conducted at each feeding station for 3 hours on three different days in September-October and similarly again in February, summing up to a total 126 hours of mistnetting. The time of day (morning/day/afternoon) was rotated for each site. Nets were checked every 30 minutes and mass $(\sim 0.01 \mathrm{~g})$, wing $(\sim 0.5 \mathrm{~mm})$ were recorded for each bird. $A$ small blood sample (40-60 $\mu$ l) was taken, kept on ice, centrifuged within 8 hours, and RBCs frozen at $-80^{\circ} \mathrm{C}$ for telomere and mitochondria density analyses.

\section{Thyroid hormones and oxidative stress assays}

Plasma thyroid hormones (triiodothyronine (T3), the biologically active form T3 and thyroxine (T4) a precursor, expressed as $\mathrm{ng} / \mathrm{mL}$ ) were measured from 14-day-old nestlings with nano-LC-MS/MS following Ruuskanen et al. (2019; 2018). Due to practical constraints, we randomly selected one to four nestling per nest, $n=13 \mathrm{TH}$ and $\mathrm{n}=11$ control nestlings for analysis.

Total glutathione (tGSH), the most abundant intra-cellular antioxidant, and the ratio between reduced and oxidized gluthatione (GSH:GSSG, an indicator of oxidative challenge) were measured from whole-blood samples with the ThioStar ${ }^{\circledR}$ Glutathione Fluorescent Detection Kit (K005-FI, Arbor Assays, USA) (Sarraude et al., 2020a). As a measure of oxidative damage, we assessed blood lipid peroxidation (malonaldehyde, MDA) using the TBARS-assay following Espin et al. (2017). Both measurements had CV\% $<10$ and are expressed per mg of protein (measured via BCA protein assay, ThermoFisher Scientific). Biomarkers of oxidative 
stress were measured from 2 randomly selected nestlings per nest $(n=33 \mathrm{TH}, \mathrm{n}=26$ control nestlings).

\section{qPCR assays for relative telomere length, mtDNA copy number and molecular sexing}

We extracted DNA from blood cells using a standard salt extraction alcohol precipitation method (Aljanabi and Martinez, 1997). Extracted DNA was diluted in elution buffer BE for DNA preservation. DNA concentration and quality $(260 / 280>1.80$ and $260 / 230$ > 2.00) were checked with a ND-1000-Spectrophotometer (NanoDrop Technologies, Wilmington, USA). DNA integrity was verified in 24 samples chosen randomly using gel electrophoresis (50 ng of DNA, $0.8 \%$ agarose gel at $100 \mathrm{mV}$ for $60 \mathrm{~min}$ ) and DNA staining with Midori Green. Each sample was then diluted to a concentration of $1.2 \mathrm{ng} \cdot \mu^{-1}$ for subsequent qPCR analysis.

Relative telomere length $(r T L)$ and mitochondrial DNA copy number (mtDNAcn, an index of mitochondrial density) were quantified using qPCR. This technique estimates relative telomere length by determining the ratio $(T / S)$ of telomere repeat copy number $(T)$ to a single copy gene (SCG), and the relative mtDNAcn as the ratio between one mitochondrial gene and the same single copy gene. Here, we used RAG1 as a SCG (verified as single copy using a BLAST analysis on the great tit genome) and cytochrome oxidase subunit 2 (COI2) as a mitochondrial gene (verified as non-duplicated in the nuclear genome using a BLAST analysis). Forward and reverse telomere primers were 5'-CGGTTTGTTTGGGTTTGGGTTTGGGTTTGGGTTTGGGTT-3' (Tel-1b) and 5'-GGCTTGCCTTACCCTTACCCTTACCCTTACCCTTACCCT-3' (Tel-2b) respectively. Forward and reverse RAG1 primers were 5'-TCGGCTAAACAGAGGTGTAAA-3' and 5'CAGCTTGGTGCTGAGATGTAT-3', respectively. Forward and reverse COI2 primers were 5'CAAAGATATCGGCACCCTCTAC-3'; 5'- GCCTAGTTCTGCACGGATAAG-3', respectively. For the 
qPCR assays, the reactions were performed on a 384-QuantStudio ${ }^{\text {TM }} 12 \mathrm{~K}$ Flex Real-Time PCR

211 System (Thermo Fisher), in a total volume of $12 \mu \mathrm{L}$ including $6 \mathrm{ng}$ of DNA, primers at a final concentration of 300nM and $6 \mu \mathrm{L}$ of SensiFAST ${ }^{\mathrm{TM}}$ SYBR lo-ROX (Bioline). Telomere, RAG1 and COI2 reactions were performed in triplicates on the same plates (10 plates in total); the qPCR conditions were: $3 \mathrm{~min}$ at $95^{\circ} \mathrm{C}$, followed by 35 cycles of $10 \mathrm{~s}$ at $95^{\circ} \mathrm{C}, 15 \mathrm{~s}$ at $58^{\circ} \mathrm{C}$ and $10 \mathrm{~s}$ at $72^{\circ} \mathrm{C}$. A DNA sample being a pool of DNA from 10 adult individuals was used as a reference sample and was included in triplicate on every plate. The efficiency of each amplicon was estimated from a standard curve of the reference sample ranging from 1.5 to $24 \mathrm{ng}$. The mean reaction efficiencies were $109.1 \pm 1.8 \%$ for telomere, $102.2 \pm 1.6 \%$ for RAG1, $96.3 \pm 1.1 \%$ for COI2. The relative telomere length and mtDNAcn of each sample were calculated as $\left(1+E f_{\text {Tel or }}\right.$ $\left.\mathrm{COI}_{2}\right)^{\Delta \mathrm{Cq} \text { Tel or COI2 }} /\left(1+E f_{\mathrm{RAG1}}\right)^{\Delta \mathrm{CqRAG1}} ; E f$ being the amplicon efficiency, and $\Delta \mathrm{Cq}$ the difference in Cq-values between the reference sample and the focal sample. Intra-plate technical repeatabilities of telomere and $m t D N A c n$ based on triplicates were 0.87 (95\% C.I. [0.85-0.89]) and 0.96 (95\% C.I. [0.95-0.97]) respectively. Inter-plate technical repeatabilities of telomere and $m t D N A c n$ based on one repeated plate were 0.98 (95\% C.I. [0.97-0.99]) and 0.77 (95\% C.I. [0.59-0.88]) respectively.

The use of $m t D N A c n$ as an index of mitochondrial density has been questioned in human (Larsen et al., 2012), but we have previously shown good correlations between mtDNAcn and mitochondrial respiration rates in pied flycatcher (Stier et al. 2019). Great tits have quite peculiar telomeres, characterized notably by some ultra-long telomeres that do not seem to shorten with age in adults(Atema et al., 2019). Since qPCR only provides an estimate of overall telomere length, it has been suggested it could be suboptimal for this study species. Yet, relative telomere length (i.e. measured using $\mathrm{qPCR}$ ) in this species has been shown to shorten during the nestling stage (Stier et al., 2016, 2015), to respond to environmental factors (e.g. hatching asynchrony: (Salmón 
et al., 2016; Stier et al., 2016, 2015) and to predict adult survival (Salmón et al., 2017). Withinindividual repeatability of telomere length has recently been suggested to be an important factor to evaluate the pertinence of telomere length data in a given study/species (Kärkkäinen et al., n.d.), and the biological repeatability in our dataset was $R=0.66$ [0.55-0.79], which is above the average reported by qPCR studies (i.e. $R=0.47$ ), and within the upper range of what has been reported for great tits (Kärkkäinen et al. 2021)

Birds were molecularly sexed using a qPCR approach adapted from(Chang et al., 2008; Ellegren and Fridolfsson, 1997). Forward and reverse sexing primers were 5'CACTACAGGGAAAACTGTAC-3' (2987F) and 5'- CCCCTTCAGGTTCTTTAAAA - $3^{\prime}$ (3112R), respectively. qPCR reactions were performed in a total volume of $12 \mu \mathrm{L}$ including $6 \mathrm{ng}$ of DNA, primers at a final concentration of $800 \mathrm{nM}$ and $6 \mu \mathrm{L}$ of SensiFAST ${ }^{\mathrm{TM}} \mathrm{SYBR}^{\circledR}$ Lo-ROX Kit (Bioline). qPCR conditions were: $3 \mathrm{~min}$ at $95^{\circ} \mathrm{C}$, followed by 40 cycles of $45 \mathrm{~s}$ at $95^{\circ} \mathrm{C}, 60 \mathrm{~s}$ at $52^{\circ} \mathrm{C}$ and $60 \mathrm{~s}$ at $72^{\circ} \mathrm{C}$, then followed by a melting curve analysis $\left(95^{\circ} \mathrm{C} 60 \mathrm{~s}, 45^{\circ} \mathrm{C} 50 \mathrm{~s}\right.$, increase to $95^{\circ} \mathrm{C}$ at $\left.0.1^{\circ} \mathrm{C} / \mathrm{s}, 95^{\circ} \mathrm{C} 30 \mathrm{~s}\right)$. Samples were run in duplicates in a single plate and 6 adults of known sex were included as positive controls. Sex was determined by looking at the dissociation curve, with two peaks indicating the presence of a $Z$ and $W$ chromosome (female), and one peak indicating the presence of only the $Z$ chromosomes (male).

\section{Statistical analyses}

We ran several linear or generalized linear mixed models (LMMs/GLMMs) for both nestling and juvenile data. In order to account for genetic effects and the effects of growing environment other than prenatal hormones, we included the IDs of both the nest of origin (i.e. where a nestling hatched) and the nest of rearing (i.e. where a nestling grew up) as 
random intercepts in all suitable models. In models with repeated measurements of the same

258

259

260

261

262 individuals, the ID of each individual was included as a random intercept and age as a random slope to interact with both the original and rearing nests, accounting for the variation in growth rate between nests. For physiological measurements, assay batch ID was included either as random intercept or fixed factors (depending on the number of levels, see Tables for details).

To analyze nestling growth, we separated the data into the early (i.e. from day 2 to day 7) and late (i.e. from day 7 to day 14) phases. Because of the start of heating treatment at day 7 , this separation allows us to make use of the larger sample size during the early phase and focus on the nestlings from non-heated nests during the late phase. The fixed factors of these models included the prenatal hormone treatment ( $\mathrm{TH}$ versus $\mathrm{CO}$ ), age, and cross-fostering (yes or no). For tarsus length, we additionally controlled for the measurer ID as a fixed factor. The potential effect of brood size (at day 2 ) was initially explored but subsequently removed from the models for simplicity because no significant effect was found. Because molecular sexing was only conducted on nestlings having DNA extracted for mtDNAcn and rTL, not all nestlings included in the growth analyses were sexed. We therefore did not include sex in order to make use of the full data set. Repeating our models with only the sexed nestlings still gave qualitatively the same results and did not show sex-specific effects of prenatal THs on growth. For the juveniles captured during autumn/winter we ran LMMs with hormone treatment, date of capture, and sex (because of clearer sexual size dimorphism at this age) as fixed factors.

For all models of physiological measurements fixed factors included prenatal hormone treatment, nestling sex and body mass. Because both mtDNAcn and rTL are ratios to a single 
copy gene, we also z-transformed the data to allow across-study comparison (Verhulst, 2020).

281 Age was included as a three-level categorical variable (day 7, day 14, and autumn) in order to estimate the changes of mtDNAcn and rTL between each time point.

We used GLMMs to estimate the influence of prenatal thyroid hormones on hatching success, fledging success (i.e. pre-fledging survival), and post-fledging survival. We modelled the outcome of each egg (i.e. hatched or not) or nestling (i.e. survived or not) using a logit link function and specifying a binomial residual distribution. For hatching success, two nests in which eggs never hatched (i.e. cause of failure likely unrelated to our treatment) were excluded, giving a final sample size of 354 eggs from 42 nests. The nest ID was treated as a random intercept, and prenatal hormone treatment and laying date of each nest as fixed factors. For fledging success, all 131 nestlings (reared in 22 nests after cross-fostering) that successfully hatched were included in the analysis. The ID of rearing nest was treated as a random intercept and prenatal hormone treatment, laying date, brood size, and crossfostering as fixed factors. For post-fledging survival, the 99 nestlings that successfully fledged were included in the analysis. The individuals that were never recaptured in the autumn were presumed dead. In this model, including nest ID as random intercepts caused convergence problem. We therefore fitted a binomial GLM instead, with prenatal hormone treatment, cross-fostering, and body mass at day 14 (i.e. the last measurement before fledging) as predictors.

In all statistical models described above, hormone-related two-way interactions were of interest and therefore included. Following the suggestion by (Schielzeth, 2010), input variables (except for the technical variables) were mean-centered before model fitting. 
Significant interactions were further examined by post-hoc interaction analysis using the $R$ package emmeans (Lenth 2021).

All LMMs and GLMMs were conducted in the R environment 3.6.1, using the package Ime4 (Bates et al., 2015) and ImerTest (Kuznetsova et al., 2017). P values were determined using the Kenward-Roger method to approximate the denominator degrees of freedom $(R$ package pbkrtest, (Halekoh and Hojsgaard, 2014), implemented within ImerTest) for LMMs and by Laplace approximation for GLMMs. Model assumptions were diagnosed by using the $R$ package DHARMa (Hartig 2021) and no clear violations were observed except for the models for plasma T3. Nevertheless, given the fact that the model of T3 did not detect any significant effect and the general robustness of LMMs (Schielzeth et al., 2020), this did not influence our conclusion.

\section{Results}

Early body mass growth (between day 2 and day 7 post-hatching) did not differ between TH-supplemented and control groups (Table 1a, Fig 1a), but individuals from THsupplemented eggs grew faster at the later nestling stage, i.e. between day 7 and day 14 posthatching (Hormonexage interaction, $F_{1,20.18}=5.423, p=0.030$, Table $1 b$, Fig $1 b$; body mass gain (mean $\pm \mathrm{SE}$ ) in TH-nestlings $0.898 \pm 0.042 \mathrm{~g} /$ day, CO-nestlings $0.785 \pm 0.043 \mathrm{~g} /$ day). Similarly, individuals from TH-supplemented eggs expressed faster tarsus growth rate between day 7 and day 14 post-hatching $\left(F_{1,32.40}=4.134, p=0.050\right.$, Table $1 c, T H: 0.567 \pm 0.032 \mathrm{~mm} /$ day, $\mathrm{CO}$ : $0.475 \pm 0.033 \mathrm{~mm} /$ day, Fig $1 \mathrm{c})$. When analyzing the body mass and tarsus separately for each age, body mass was slightly smaller on day 2 and day 7 in the TH-supplemented group compared to control, yet there was no statistically significant difference between the groups 
bioRxiv preprint doi: https://doi.org/10.1101/2021.12.22.473794; this version posted December 22,2021 . The copyright holder for this preprint (which was not certified by peer review) is the author/funder, who has granted bioRxiv a license to display the preprint in perpetuity. It is made available under aCC-BY-NC-ND 4.0 International license.

325 at any age (Fig 1, Table S1). In juveniles, there was no difference in body mass or wing length

326 between the treatment groups (Body mass: $F_{1,7.24}=0.012, p=0.916$; wing length: $F_{1,11.48}=$ $327 \quad 0.005, p=0.948)$. 


\begin{tabular}{|c|c|c|c|c|c|c|}
\hline \multicolumn{7}{|c|}{ (a) Early nestling growth (day 2 - day 7 ) } \\
\hline Random effects: & & Variance & Std. Dev. & & & \\
\hline Individual ID (n=224) & Intercept & 0.843 & 0.918 & & & \\
\hline \multirow{2}{*}{ Nest of origin $(n=42)$} & Intercept & 0.628 & 0.793 & & & \\
\hline & Age & 0.005 & 0.070 & & & \\
\hline \multirow[t]{2}{*}{ Nest of rearing $(n=42)$} & Intercept & 0.057 & 0.239 & & & \\
\hline & Age & 0.007 & 0.083 & & & \\
\hline Residual & & 0.203 & 0.450 & & & \\
\hline Fixed factors & Estimate & SE & $\mathbf{t}$ & df & $\mathbf{F}$ & $p$ \\
\hline Intercept & 8.229 & 0.146 & 56.516 & & & \\
\hline Hormone (TH) & -0.181 & 0.142 & -1.277 & $1,38.27$ & 1.632 & 0.209 \\
\hline Age & 1.730 & 0.019 & 89.617 & $1,45.65$ & 8031.211 & $<0.001$ \\
\hline Cross-foster (yes) & -0.139 & 0.069 & -2.006 & $1,187.94$ & 4.025 & 0.046 \\
\hline Hormone $\times$ Age & 0.009 & 0.016 & 0.573 & 1. 28.88 & 0.328 & 0.571 \\
\hline Hormone $\times$ Cross-foster & 0.037 & 0.076 & 0.485 & $1,34.61$ & 0.235 & 0.631 \\
\hline
\end{tabular}

(b) Late nestling body mass growth (day 7 - day 14)

\begin{tabular}{|c|c|c|c|c|c|c|}
\hline Random effects: & & Variance & Std. Dev. & & & \\
\hline Individual ID (n=111) & Intercept & 0.567 & 0.753 & & & \\
\hline \multirow{2}{*}{ Nest of origin $(n=36)$} & Intercept & 0.381 & 0.617 & & & \\
\hline & Age & 0.007 & 0.084 & & & \\
\hline \multirow[t]{2}{*}{ Nest of rearing $(n=20)$} & Intercept & 0.003 & 0.058 & & & \\
\hline & Age & 0.015 & 0.120 & & & \\
\hline Residual & & 0.534 & 0.731 & & & \\
\hline Fixed factors & Estimate & SE & $\mathbf{t}$ & df & $\mathbf{F}$ & $\mathbf{p}$ \\
\hline Intercept & 15.496 & 0.152 & 101.728 & & & \\
\hline Hormone (TH) & -0.240 & 0.310 & -0.774 & $1,29.58$ & 0.598 & 0.445 \\
\hline Age & 0.841 & 0.035 & 24.228 & $1,19.41$ & 586.992 & $<0.001$ \\
\hline Cross-foster (yes) & -0.100 & 0.269 & -0.686 & $1,32.46$ & 0.138 & 0.713 \\
\hline Hormone $\times$ Age & 0.113 & 0.049 & 1.853 & $1,20.18$ & 5.423 & 0.030 \\
\hline Hormone $\times$ Cross-foster & 0.321 & 0.643 & 0.500 & $1,20.30$ & 0.250 & 0.623 \\
\hline
\end{tabular}

(c) Late nestling tarsus growth (day 7 - day 14)

\begin{tabular}{lrrrrrr} 
Random effects: & & Variance & Std. Dev. & & & \\
\hline Individual ID (n=111) & Intercept & 0.239 & 0.489 & & & \\
Nest of origin (n=36) & Intercept & 0.221 & 0.470 & & & \\
& Age & 0.012 & 0.111 & & & \\
Residual & & 0.376 & 0.613 & & & \\
\hline Fixed factors & Estimate & \multicolumn{1}{c}{ SE } & \multicolumn{1}{c}{$\mathbf{t}$} & df & $\mathbf{F}$ & \\
\hline Intercept & 20.607 & 0.168 & 122.819 & & & \\
Hormone (TH) & -0.126 & 0.216 & -0.583 & $1,34.02$ & 0.340 & 0.563 \\
Age & 0.520 & 0.023 & 22.379 & $1,36.42$ & 500.810 & $<0.001$ \\
Cross-foster (yes) & -0.141 & 0.171 & -0.824 & $1,51.71$ & 0.679 & 0.414 \\
Measurer 2 & 0.235 & 0.180 & 1.306 & $2,122.11$ & 7.626 & $<0.001$ \\
Measurer 3 & 0.601 & 0.169 & 3.551 & & & \\
Hormone $\times$ Age & 0.092 & 0.045 & 2.033 & $1,32.40$ & 4.134 & $\mathbf{0 . 0 5 0}$ \\
Hormone $\times$ Cross-foster & 0.436 & 0.342 & 1.276 & $1,52.56$ & 1.628 & 0.208 \\
\hline
\end{tabular}

Sample sizes: 
Early growth, $\mathrm{n}=99$ control and $119 \mathrm{TH}$-supplemented at day 2, 104 control and

117 TH-supplemented at day 7

Late growth, $\mathrm{n}=57$ control and $54 \mathrm{TH}$-supplemented at day 7, 50 control and

49 TH-supplemented at day 14

(a)

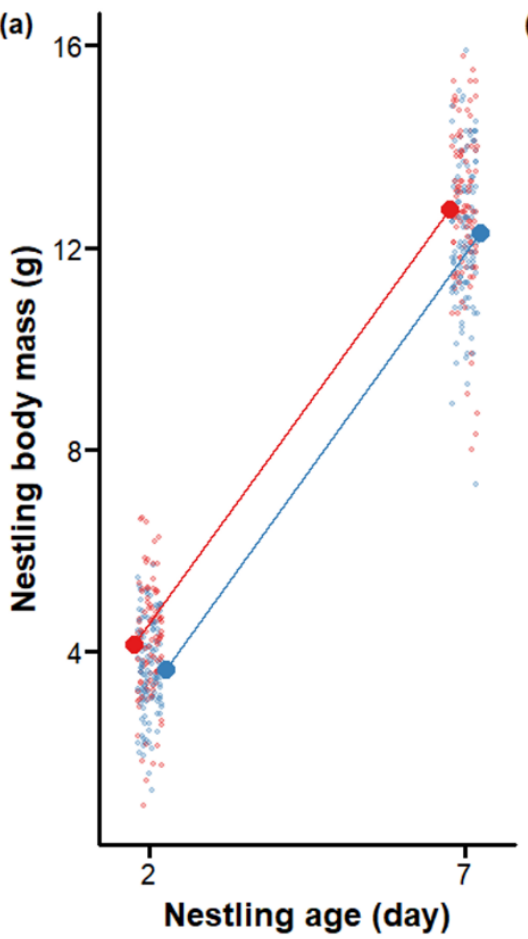

(b)

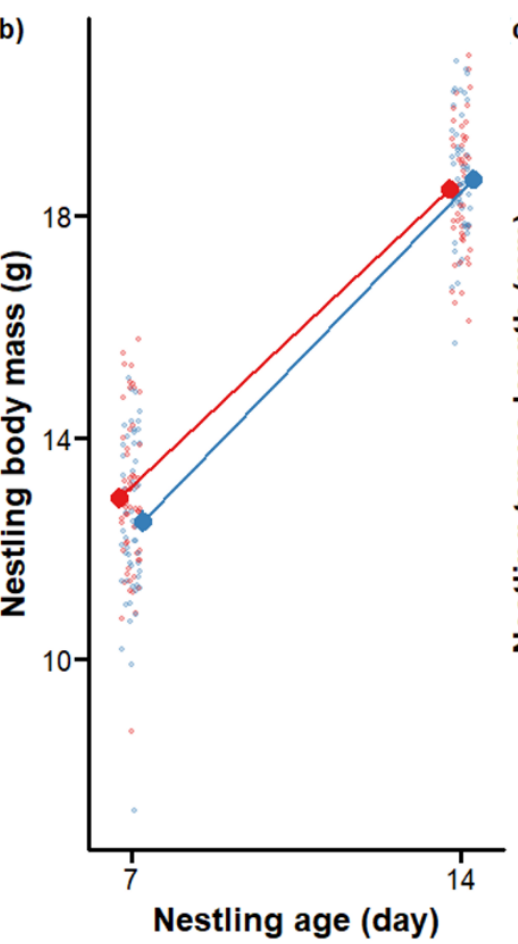

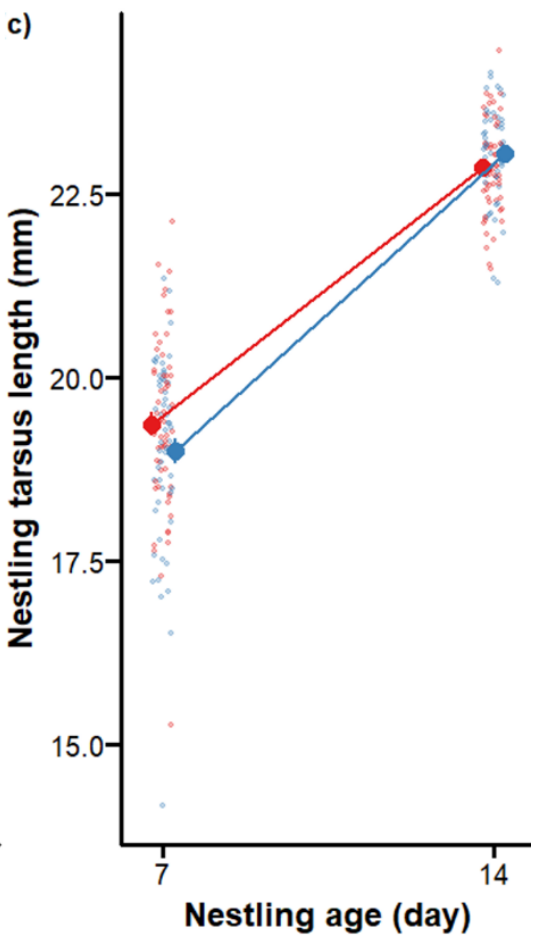

Fig. 1. Nestling growth in prenatally TH-supplemented (blue dots) and control (red dots) 

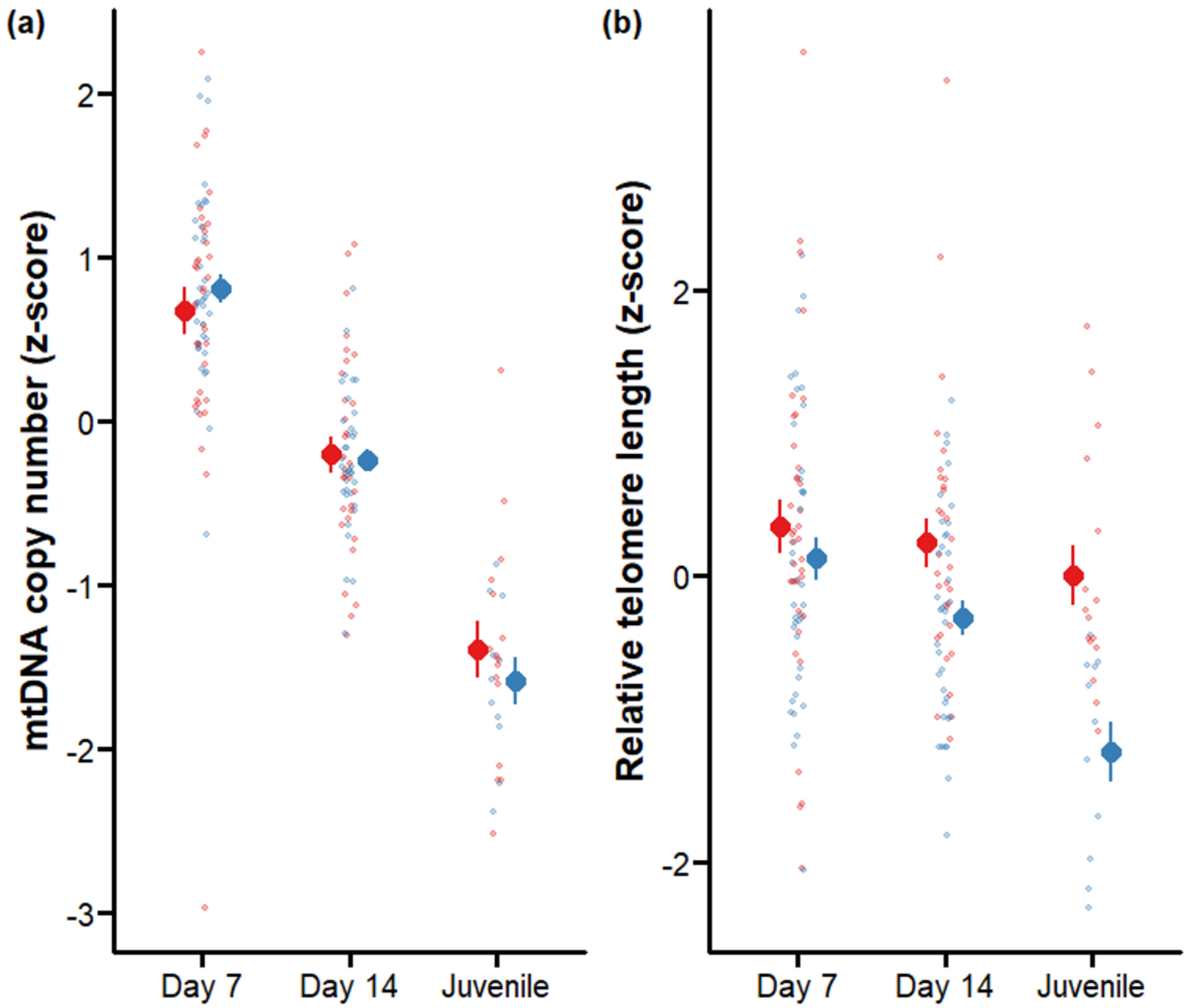

Fig 2. (a) Relative mitochondrial density (z-scale) and (b) relative telomere length in prenatally

TH-supplemented (blue dots) and control groups (red dots) at different developmental stages

scatter of the raw data (small, semi-transparent dots) are shown. See sample sizes in the text.

Biomarkers of intracellular oxidative status (total GSH, GSH:GSSG ratio) and damage to lipids

14 days post-hatching did not clearly differ among the treatment groups (marginal means \pm SE:

ratio: TH-supplemented, $0.051 \pm 0.008$, control, $0.065 \pm 0.011$; MDA nmol/mg protein: $\mathrm{TH}$ - 
supplemented, 0.038 \pm 0.005 , control, 0.037 \pm 0.005 ; Table S2). Plasma T3 and T4 also did not were not associated with body mass or sex ( $p>0.06$, Table S3)

Mitochondrial density decreased with age, but there were no clear differences between prenatally TH-supplemented and control groups at any age (d7, d14 or juveniles, Fig 2a, Table 2). Post-hoc analyses indicated significant decrease between each age category (all Tukeyadjusted $p<0.001$ ), regardless of the hormone treatment. Mitochondrial density was not associated with sex or body mass (Table 2).

Tukey post-hoc test: $\mathrm{TH}$ vs control: $\mathrm{t}_{40.6}=1.357, \mathrm{p}=0.182$ ). Telomeres were significantly $\left.t_{44.1}=2.548, p=0.014\right)$, and this difference was accentuated in juveniles, offspring from $\mathrm{TH}$ 
372 individuals (TH 67.6\%, control 69.1\%; $z=0.314, p=0.754$ ). Nestling survival to fledging was

373 marginally, but not statistically significantly higher in differ prenatally TH supplemented than

374 and control nestlings ( $\mathrm{TH}: 83.05 \%$ vs $\mathrm{CO} 69.44 \%, z=0.56, p=0.575)$. Fledging success was not

375 associated with laying date or brood size $(p>0.6)$. Juvenile recapture rate was not significantly

376 different across the groups (TH: $28.57 \%$ vs CO $34.00 \%, z=-0.46, p=0.645$ ).

377 Table 2. Linear mixed models of mitochondrial density and telomere length in blood cells

378 growth in response to prenatal TH-supplementation.

\begin{tabular}{|c|c|c|c|c|c|c|}
\hline \multicolumn{7}{|l|}{ (a) Mitochondria density } \\
\hline Random effects: & & Variance & Std. Dev. & & & \\
\hline Individual ID (n=78) & Intercept & 0.088 & 0.297 & & & \\
\hline Nest of origin $(n=35)$ & Intercept & 0.044 & 0.211 & & & \\
\hline Nest of rearing $(n=19)$ & Intercept & $<0.001$ & $<0.001$ & & & \\
\hline DNA batch $(n=8)$ & Intercept & 0.022 & 0.148 & & & \\
\hline Residual & & 0.238 & 0.488 & & & \\
\hline Fixed factors & Estimate & SE & $\mathbf{t}$ & df & $\mathbf{F}$ & $\mathbf{p}$ \\
\hline Intercept & -0.017 & 0.086 & -0.198 & & & \\
\hline Hormone (TH) & -0.014 & 0.140 & -0.098 & $1,23.49$ & 0.010 & 0.923 \\
\hline Sex & 0.225 & 0.128 & 1.756 & $1,67.12$ & 3.083 & 0.084 \\
\hline Age day 7 & 2.196 & 0.121 & 18.216 & $1,107.26$ & 331.826 & $<0.001$ \\
\hline Age day 14 & 1.227 & 0.122 & 10.042 & $1,107.07$ & 100.849 & $<0.001$ \\
\hline Day-7 body mass & -0.069 & 0.068 & -1.015 & $1,64.04$ & 1.030 & 0.314 \\
\hline Hormone $\times$ Sex & -0.035 & 0.261 & -0.134 & $1,67.03$ & 0.018 & 0.894 \\
\hline Hormone $\times$ Age day 7 & 0.363 & 0.240 & 1.518 & $1,108.03$ & 2.305 & 0.132 \\
\hline Hormone $\times$ Age day 14 & 0.196 & 0.242 & 0.808 & $1,107.99$ & 0.652 & 0.421 \\
\hline Hormone $\times$ Body mass & 0.003 & 0.136 & 0.026 & $1,66.85$ & 0.001 & 0.980 \\
\hline Sex $\times$ Age day 7 & 0.370 & 0.241 & 1.532 & $1,106.83$ & 2.347 & 0.129 \\
\hline Sex $\times$ Age day 14 & 0.076 & 0.245 & 0.311 & $1,106.76$ & 0.097 & 0.757 \\
\hline
\end{tabular}

\section{(b) relative telomere length}

Random effects:

Variance Std. Dev.

\begin{tabular}{|c|c|c|c|c|c|c|}
\hline Individual ID (n=77) & Intercept & 0.090 & 0.301 & & & \\
\hline Nest of origin $(n=35)$ & Intercept & 0.028 & 0.167 & & & \\
\hline Nest of rearing $(n=19)$ & Intercept & 0.057 & 0.238 & & & \\
\hline DNA batch $(n=8)$ & Intercept & 0.249 & 0.499 & & & \\
\hline Residual & & 0.518 & 0.720 & & & \\
\hline Fixed factors & Estimate & SE & $\mathbf{t}$ & df & $\mathbf{F}$ & p \\
\hline Intercept & -0.086 & 0.202 & -0.427 & & & \\
\hline Hormone (TH) & -0.550 & 0.174 & -3.168 & $1,18.03$ & 10.035 & 0.005 \\
\hline Sex & -0.318 & 0.168 & -1.890 & $1,60.80$ & 3.571 & 0.064 \\
\hline Age day 7 & 0.909 & 0.176 & 5.175 & $1,113.09$ & 26.778 & $<0.001$ \\
\hline
\end{tabular}




\begin{tabular}{lrrrrrr} 
Age day 14 & 0.341 & 0.092 & 3.726 & $1,112.25$ & 13.886 & $<\mathbf{0 . 0 0 1}$ \\
Day-7 body mass & 0.100 & 0.090 & 1.116 & $1,63.08$ & 1.246 & 0.268 \\
Hormone $\times$ Sex & 0.087 & 0.334 & 0.262 & $1,58.01$ & 0.069 & 0.794 \\
Hormone $\times$ Age day 7 & 0.992 & 0.347 & 2.859 & $1,116.76$ & 8.172 & $\mathbf{0 . 0 0 5}$ \\
Hormone $\times$ Age day 14 & 0.708 & 0.351 & 2.018 & $1,114.95$ & 4.074 & $\mathbf{0 . 0 4 6}$ \\
Hormone $\times$ Body mass & -0.149 & 0.175 & -0.847 & $1,62.53$ & 0.718 & 0.400 \\
Sex $\times$ Age day 7 & -0.231 & 0.351 & -0.657 & $1,113.82$ & 0.432 & 0.513 \\
Sex $\times$ Age day 14 & -0.495 & 0.355 & -1.396 & $1,111.55$ & 1.950 & 0.165 \\
\hline
\end{tabular}

Sample sizes: $n=37$ control and $41 \mathrm{TH}$-supplemented 


\section{Discussion}

By manipulating prenatal exposure to THs in a wild passerine species, we demonstrate that an increase in prenatal THs can accelerate both postnatal growth and telomere shortening. Yet, we did not detect significant effects of elevated prenatal THs on postnatal oxidative stress levels, cellular energetics measured as mtDNA copy number, circulating TH levels, or short to medium-term survival (i.e. hatching success, fledging success, and apparent survival to the next autumn/winter). While previous findings suggested potential sexdependent effects of prenatal THs in the same species (Ruuskanen et al., 2016a), our results do not support such a hypothesis.

Shorter telomeres in the TH-supplemented group were only detected from 14 days after hatching onwards, which seems to exclude direct effects or prenatal TH on telomere dynamics during embryonic development. Yet, this coincides with the accelerated postnatal body mass and tarsus growth observed in the TH-group (between day 7 and 14) compared to controls, and faster growth can accelerate telomere shortening either through enhanced cell division or through inducing oxidative stress (Monaghan and Ozanne, 2018). Considering the lack of impact of prenatal THs on oxidative stress reported here and in previous work on birds (Hsu et al., 2020, 2019a; Sarraude et al., 2020a; Sarraude et al., 2020b), it seems unlikely that the accelerated telomere shortening observed in this study would be linked to increased oxidative damage on DNA. Yet, we cannot fully rule out this explanation since DNA damage was not directly assessed and oxidative stress sensitivity might vary between different biomolecules (Reichert and Stier, 2017). The effect of prenatal THs on telomere length seemed to increase with age (i.e. stronger effect observed in juveniles than at day 14), which could be explained by a delayed effect of fast postnatal growth (day 7 to day 14) because telomeres 
only shorten at the subsequent cellular division, and therefore delayed effects are likely to be observed (Monaghan and Ozanne, 2018). Our hypothesis that prenatal THs could program postnatal metabolic and endocrine function (and thus affect telomere shortening indirectly) was not supported as we found no evidence for differences in mitochondrial density or plasma TH levels (key coordinators of metabolism) across the treatment groups. The latter results are not fully surprising considering the limited evidence supporting a prenatal programming of plasma THs (sex-specific effect on T4 only, Hsu et al. 2017; no effect, Hsu et al. 2020) or mitochondrial density (no effect, Stier et al. 2020; Hsu et al. 2020). According to the metabolic telomere attrition hypothesis, telomere shortening is likely to be increased during energydemanding periods, and accelerated growth under limited resources is likely carrying a metabolic cost (Casagrande and Hau, 2019).

The effects observed here on telomeres in response to increased prenatal TH levels are in sharp contrast with our previous findings in another passerine species, the collared flycatcher (Stier et al. 2020). Indeed, increasing prenatal THs in the collared flycatcher increased telomere length measured very shortly after hatching (day 2), while not affecting telomere shortening during postnatal growth (Stier et al. 2020). There are several alternative explanations for these contrasted findings: (1) we cannot exclude that prenatal THs increased telomere length during embryonic development in great tits since our first telomere length measurement (day $7, \mathrm{ca} .70 \%$ of fledging body mass) was done considerably later than in collared flycatchers (i.e. day 2, ca. $20 \%$ of fledging body mass). (2) THs differently influenced post-natal growth in the two species: great tits from the TH-supplemented eggs were initially slightly smaller, but grew faster in the late nestling period, whereas collared flycatchers from the TH-supplemented eggs were bigger soon after hatching, but grew slightly slower during the nestling stage (Hsu et al., 2019a). These contrasting growth patterns may explain, at least 
partly, the contrasted findings in these two species: an increased metabolic demand for fast

428

429

430

431

432

postnatal growth in great tit in response to prenatal TH could accelerate telomere shortening, while a reduced metabolic demand in collared flycatcher from $\mathrm{TH}$-injected eggs could enable telomere maintenance (Casagrande and Hau, 2019). Measurements of the mTOR signaling pathway could shed light on the validity of this hypothesis (Casagrande and Hau, 2019). (3) The effects of maternal signals on offspring are expected to differ based on environmental conditions, so called context-dependent effects: For example, prenatal androgens have been found to differentially influence offspring growth depending on season or food availability (Groothuis et al., 2020; Muriel et al., 2015). We recently found no evidence for prenatal THs differentially influencing growth and early-life survival depending on rearing temperature (Hsu et al. 2020), yet the influence of resource availability was not tested. In two sister species, collared and pied flycatchers, differential effects of prenatal THs on growth may have been explained by differences in food resources (Sarraude et al. 2020b). (4) These two species exhibit different life-histories, as collared flycatchers are migratory and great tits (relatively) sedentary. Migratory species have generally higher metabolism (Jetz et al., 2008) as well as an overall faster pace of life (Soriano-Redondo et al. 2021), and may thus present differences in TH physiology. We may speculate that the telomere maintenance, and role of THs in the regulation of telomere maintenance may differ across species with different life-histories. Telomere maintenance is known to be influenced by e.g. species lifespan (Haussmann et al., 2004) and migratory populations within a species have shorter telomeres (Bauer et al., 2016). Yet, to our knowledge, studies have not considered migratory vs. non-migratory species in telomere maintenance. Species-specific effects of the prenatal hormonal environment on telomere dynamics have already been described: elevated prenatal glucocorticoid levels led to shorter telomeres in domestic chicken and female zebra finch (Haussmann et al., 2012; 
Tissier et al., 2014), but to longer telomeres in yellow-legged gull (Noguera, da Silva, and Velando 2020). To understand if any of these hypotheses is likely to explain the contrasted effects found here and in Stier et al. (2020), more studies on the impact of prenatal THs on telomere biology across taxa are needed.

To understand the selective pressures on maternal allocation and signaling, it is important to characterize true fitness effects from development to survival and lifetime reproductive success. In contrast to our predictions, we found no effects of elevated prenatal TH-supplementation on apparent survival or individual condition during their first autumn/ winter. Unfortunately, longer-term effects on survival and reproductive success are very challenging to measure in such an experimental setting in the wild, as the recruitment to first breeding is usually very low (e.g. < 10\% Radersma et al., 2015), which requires a very high number of nests to be manipulated, something often not feasible for both logistical and ethical reasons. Yet, long-term effects of accelerated early-life telomere shortening may lead to a decrease in longer-term survival and lifespan (Eastwood et al., 2019; Heidinger et al., 2012). The negative effects on later stages suggest that there need to be benefits of high prenatal THs for the offspring or mother, potentially during early-life stages, where selection can be strong. In this study, the elevated growth rates could increase offspring competitive abilities early in life, yet, the effects of prenatal THs on growth seem to be highly inconsistent across studies (Hsu et al., 2020, 2019a, 2017; Ruuskanen et al., 2016a; Sarraude et al., 2020a; Sarraude et al., 2020b). While circulating THs are known to be associated with health and ageing in humans and mammalian models (Bano et al. 2017, 2019; Møllehave et al. 2020), our study is the first to show that exposure to higher prenatal thyroid hormones can lead to accelerated cellular ageing measured through telomere length. This should stimulate further 
research using both epidemiological and experimental approaches across taxa to uncover the potential regulation of telomere biology by thyroid hormones both pre- and postnatally.

\section{Author contribution}

BYH, AS \& SR designed the study and collected field data. NCS, AS \& SR conducted laboratory work. BYH analyzed the data. BYH, AS \& SR wrote the manuscript, with input from NCS.

\section{Acknowledgements}

This study was financially supported by the Academy of Finland (\#286278 to SR). NCS acknowledges support from the EDUFI Fellowship and Maupertuis Grant. B-Y.H work was supported by a grant from the Ella and Georg Ehrnrooth Foundation and Academy of Finland. AS was supported by a 'Turku Collegium for Science and Medicine' Fellowship and a Marie Sklodowska-Curie Postdoctoral Fellowship (\#894963) at the time of writing. We thank all field assistants, especially Lucas Bousseau, Thomas Rosille, and Jorma Nurmi for their great effort. All authors declare no conflict of interest.

\section{Data accessibility}

All data will be archived and available in Figshare upon acceptance.

\section{References}

Aljanabi, S.M., Martinez, I., 1997. Universal and rapid salt-extraction of high quality genomic DNA for PCR-based techniques. Nucleic Acids Research. https://doi.org/10.1093/nar/25.22.4692 Arbeev, K.G., Verhulst, S., Steenstrup, T., Kark, J.D., Bagley, O., Kooperberg, C., Reiner, A.P., Hwang, S.-J., Levy, D., Fitzpatrick, A.L., Christensen, K., Yashin, A.I., Aviv, A., 2020. Association of Leukocyte Telomere Length With Mortality Among Adult Participants in 3 Longitudinal Studies. JAMA Netw Open 3, e200023. https://doi.org/10.1001/jamanetworkopen.2020.0023 
Atema, E., Mulder, E., van Noordwijk, A.J., Verhulst, S., 2019. Ultralong telomeres shorten with age in nestling great tits but are static in adults and mask attrition of short telomeres. Mol Ecol Resour 19, 648-658. https://doi.org/10.1111/1755-0998.12996

Bano, A., Chaker, L., Mattace-Raso, F.U.S., Terzikhan, N., Kavousi, M., Ikram, M.A., Peeters, R.P., Franco, O.H., 2019. Thyroid function and life expectancy with and without noncommunicable diseases: A population-based study. PLoS Med 16, e1002957. https://doi.org/10.1371/journal.pmed.1002957

Bano, A., Dhana, K., Chaker, L., Kavousi, M., Ikram, M.A., Mattace-Raso, F.U.S., Peeters, R.P., Franco, O.H., 2017. zeta Association of Thyroid Function With Life Expectancy With and Without Cardiovascular Disease The Rotterdam Study. Jama Internal Medicine. https://doi.org/10.1001/jamainternmed.2017.4836

Barnes, S.K., Ozanne, S.E., 2011. Pathways linking the early environment to long-term health and lifespan. Progress in Biophysics and Molecular Biology 106, 323-336. https://doi.org/10.1016/j.pbiomolbio.2010.12.005

Bates, D., Machler, M., Bolker, B.M., Walker, S.C., 2015. Fitting Linear Mixed-Effects Models Using Ime4. Journal of Statistical Software. https://doi.org/10.18637/jss.v067.i01

Bauer, C.M., Heidinger, B.J., Ketterson, E.D., Greives, T.J., 2016. A migratory lifestyle is associated with shorter telomeres in a songbird (Junco hyemalis). Auk. https://doi.org/10.1642/auk-1656.1

Casagrande, S., Hau, M., 2019. Telomere attrition: metabolic regulation and signalling function? Biology Letters. https://doi.org/10.1098/rsbl.2018.0885

Chang, H.W., Cheng, C.A., Gu, D.L., Chang, C.C., Su, S.H., Wen, C.H., Chou, Y.C., Chou, T.C., Yao, C.T., Tsai, C.L., Cheng, C.C., 2008. High-throughput avian molecular sexing by SYBR green-based real-time PCR combined with melting curve analysis. Bmc Biotechnology. https://doi.org/10.1186/1472-6750-8-12

Cioffi, F., Senese, R., Lanni, A., Goglia, F., 2013. Thyroid hormones and mitochondria: With a brief look at derivatives and analogues. Molecular and Cellular Endocrinology. https://doi.org/10.1016/j.mce.2013.06.006

Criscuolo, F., Torres, R., Zahn, S., Williams, T.D., 2020. Telomere dynamics from hatching to sexual maturity and maternal effects in the 'multivariate egg.' Journal of Experimental Biology 223, jeb232496. https://doi.org/10.1242/jeb.232496

Eastwood, J.R., Hall, M.L., Teunissen, N., Kingma, S.A., Aranzamendi, N.H., Fan, M., Roast, M., Verhulst, S., Peters, A., 2019. Early-life telomere length predicts lifespan and lifetime reproductive success in a wild bird. Molecular Ecology. https://doi.org/10.1111/mec.15002

Ellegren, H., Fridolfsson, A.K., 1997. Male-driven evolution of DNA sequences in birds. Nat Genet 17, 182-184. https://doi.org/10.1038/ng1097-182

Entringer, S., de Punder, K., Buss, C., Wadhwa, P.D., 2018. The fetal programming of telomere biology hypothesis: an update. Philos Trans R Soc Lond B Biol Sci 373, 20170151. https://doi.org/10.1098/rstb.2017.0151

Espin, S., Sanchez Virosta, P., García-Fernández, A., Eeva, T., 2017. A microplate adaptation of the thiobarbituric acid reactive substances assay to determine lipid peroxidation fluorometrically in small sample volumes. Revista de Toxicologia 34, In press.

Godfrey, K.M., Barker, D.J., 2001. Fetal programming and adult health. Public Health Nutr 4, 611624. https://doi.org/10.1079/phn2001145

Groothuis, T.G.G., Kumar, N., Hsu, B.Y., 2020. Explaining discrepancies in the study of maternal effects: the role of context and embryo. Current Opinion in Behavioral Sciences. https://doi.org/10.1016/j.cobeha.2020.10.006

Halekoh, U., Hojsgaard, S., 2014. Kenward-Roger Approximation and Parametric Bootstrap Methods for Tests in Linear Mixed Models - The R Package pbkrtest. Journal of Statistical Software.

Hartig, F. (2021). DHARMa: Residual Diagnostics for Hierarchical (Multi-Level / Mixed) Regression Models. R package version 0.4.3. https://CRAN.R-project.org/package=DHARMa 
Haussmann, M.F., Longenecker, A.S., Marchetto, N.M., Juliano, S.A., Bowden, R.M., 2012. Embryonic exposure to corticosterone modifies the juvenile stress response, oxidative stress and telomere length. Proceedings of the Royal Society B-Biological Sciences. https://doi.org/10.1098/rspb.2011.1913

Haussmann, M.F., Winkler, D.W., Huntington, C.E., Nisbet, I.C.T., Vleck, C.M., 2004. Telomerase expression is differentially regulated in birds of differing life span. Strategies for Engineered Negligible Senescence: Why Genuine Control of Aging May Be Foreseeable. https://doi.org/10.1196/annals.1297.029

Heidinger, B.J., Blount, J.D., Boner, W., Griffiths, K., Metcalfe, N.B., Monaghan, P., 2012. Telomere length in early life predicts lifespan. Proceedings of the National Academy of Sciences 109, 1743-1748. https://doi.org/10.1073/pnas.1113306109

Hsu, B.Y., Dijkstra, C., Darras, V.M., de Vries, B., Groothuis, T.G.G., 2017. Maternal thyroid hormones enhance hatching success but decrease nestling body mass in the rock pigeon (Columba livia). General and Comparative Endocrinology. https://doi.org/10.1016/j.ygcen.2016.10.011

Hsu, B.Y., Doligez, B., Gustafsson, L., Ruuskanen, S., 2019a. Transient growth-enhancing effects of elevated maternal thyroid hormones at no apparent oxidative cost during early postnatal period. Journal of Avian Biology. https://doi.org/10.1111/jav.01919

Hsu, B.Y., Sarraude, T., Cossin-Sevrin, N., Crombecque, M., Stier, A., Ruuskanen, S., 2020. Testing for context-dependent effects of prenatal thyroid hormones on offspring survival and physiology: an experimental temperature manipulation. Scientific Reports. https://doi.org/10.1038/s41598-020-71511-y

Hsu, B.Y., Verhagen, I., Gienapp, P., Darras, V.M., Visser, M.E., Ruuskanen, S., 2019b. Between- and Within-Individual Variation of Maternal Thyroid Hormone Deposition in Wild Great Tits (Parus major). American Naturalist. https://doi.org/10.1086/704738

Jetz, W., Freckleton, R.P., McKechnie, A.E., 2008. Environment, Migratory Tendency, Phylogeny and Basal Metabolic Rate in Birds. Plos One. https://doi.org/10.1371/journal.pone.0003261

Kärkkäinen, T., Briga, M., Laaksonen, T., Stier, A., n.d. Within-individual repeatability in telomere length: A meta-analysis in nonmammalian vertebrates. Molecular Ecology n/a. https://doi.org/10.1111/mec.16155

Kuznetsova, A., Brockhoff, P.B., Christensen, R.H.B., 2017. ImerTest Package: Tests in Linear Mixed Effects Models. Journal of Statistical Software 82, 1-26. https://doi.org/10.18637/jss.v082.i13

Larsen, S., Nielsen, J., Hansen, C.N., Nielsen, L.B., Wibrand, F., Stride, N., Schroder, H.D., Boushel, R., Helge, J.W., Dela, F., Hey-Mogensen, M., 2012. Biomarkers of mitochondrial content in skeletal muscle of healthy young human subjects. J Physiol 590, 3349-3360. https://doi.org/10.1113/jphysiol.2012.230185

Length, R.V. (2021). emmeans: Estimated Marginal Means, aka Least-Squares Means. R package version 1.6.2-1. https://CRAN.R-project.org/package=emmeans

Liu, J.S., Chen, Y.Q., Li, M., 2006. Thyroid hormones increase liver and muscle thermogenic capacity in the little buntings (Emberiza pusilla). Journal of Thermal Biology. https://doi.org/10.1016/j.jtherbio.2006.01.002

López-Otín, C., Blasco, M.A., Partridge, L., Serrano, M., Kroemer, G., 2013. The Hallmarks of Aging. Cell 153, 1194-1217. https://doi.org/10.1016/j.cell.2013.05.039

Marchetto, N.M., Glynn, R.A., Ferry, M.L., Ostojic, M., Wolff, S.M., Yao, R., Haussmann, M.F., 2016. Prenatal stress and newborn telomere length. American Journal of Obstetrics and Gynecology 215, 94.e1-94.e8. https://doi.org/10.1016/j.ajog.2016.01.177

McNabb, F.M.A., 2007. The hypothalamic-pituitary-thyroid (HPT) axis in birds and its role in bird development and reproduction. Critical Reviews in Toxicology. https://doi.org/10.1080/10408440601123552

Medici, M., Timmermans, S., Visser, W., Keizer-Schrama, S., Jaddoe, V.W.W., Hofman, A., Hooijkaas, H., de Rijke, Y.B., Tiemeier, H., Bongers-Schokking, J.J., Visser, T.J., Peeters, R.P., Steegers, E.A.P., 2013. Maternal Thyroid Hormone Parameters during Early Pregnancy and Birth 
Weight: The Generation R Study. Journal of Clinical Endocrinology \& Metabolism. https://doi.org/10.1210/jc.2012-2420

Metcalfe, N.B., Monaghan, P., 2001. Compensation for a bad start: grow now, pay later? Trends in Ecology \& Evolution.

Møllehave, L.T., Skaaby, T., Linneberg, A., Knudsen, N., Jørgensen, T., Thuesen, B.H., 2020. The association of thyroid stimulation hormone levels with incident ischemic heart disease, incident stroke, and all-cause mortality. Endocrine 68, 358-367. https://doi.org/10.1007/s12020-020-02216-5

Monaghan, P., Ozanne, S.E., 2018. Somatic growth and telomere dynamics in vertebrates: relationships, mechanisms and consequences. Philos Trans R Soc Lond B Biol Sci 373, 20160446. https://doi.org/10.1098/rstb.2016.0446

Mullur, R., Liu, Y.-Y., Brent, G.A., 2014. Thyroid Hormone Regulation of Metabolism. Physiological Reviews 94, 355-382. https://doi.org/10.1152/physrev.00030.2013

Muñoz-Lorente, M.A., Cano-Martin, A.C., Blasco, M.A., 2019. Mice with hyper-long telomeres show less metabolic aging and longer lifespans. Nat Commun 10, 4723. https://doi.org/10.1038/s41467-019-12664-x

Muriel, J., Salmon, P., Nunez-Buiza, A., de Salas, F., Perez-Rodriguez, L., Puerta, M., Gil, D., 2015. Context-dependent effects of yolk androgens on nestling growth and immune function in a multibrooded passerine. Journal of Evolutionary Biology. https://doi.org/10.1111/jeb.12668

Noguera, J.C., da Silva, A., Velando, A., n.d. Egg corticosterone can stimulate telomerase activity and promote longer telomeres during embryo development. Molecular Ecology n/a. https://doi.org/10.1111/mec.15694

Parolini, M., Possenti, C.D., Caprioli, M., Rubolini, D., Romano, A., Saino, N., 2019. Egg Testosterone Differentially Affects Telomere Length in Somatic Tissues of Yellow-Legged Gull Embryos. Physiological and Biochemical Zoology. https://doi.org/10.1086/705037

Podmokla, E., Drobniak, S.M., Rutkowska, J., 2018. Chicken or egg? Outcomes of experimental manipulations of maternally transmitted hormones depend on administration method - a meta-analysis. Biological Reviews. https://doi.org/10.1111/brv.12406

Radersma, R., Komdeur, J., Tinbergen, J.M., 2015. Early morning fledging improves recruitment in Great Tits Parus major. Ibis 157, 351-355. https://doi.org/10.1111/ibi.12230

Reichert, S., Stier, A., 2017. Does oxidative stress shorten telomeres in vivo? A review. Biology Letters. https://doi.org/10.1098/rsbl.2017.0463

Ruuskanen, S., Darras, V.M., Visser, M.E., Groothuis, T.G.G., 2016a. Effects of experimentally manipulated yolk thyroid hormone levels on offspring development in a wild bird species. Hormones and Behavior. https://doi.org/10.1016/j.yhbeh.2016.03.006

Ruuskanen, S., Espin, S., Sanchez-Virosta, P., Sarraude, T., Hsu, B.Y., Pajunen, P., Costa, R.A., Eens, M., Hargitai, R., Torok, J., Eeva, T., 2019. Transgenerational endocrine disruption: Does elemental pollution affect egg or nestling thyroid hormone levels in a wild songbird? Environmental Pollution. https://doi.org/10.1016/j.envpol.2019.01.088

Ruuskanen, S., Groothuis, T.G.G., Schaper, S.V., Darras, V.M., de Vries, B., Visser, M.E., 2016b. Temperature-induced variation in yolk androgen and thyroid hormone levels in avian eggs. General and Comparative Endocrinology. https://doi.org/10.1016/j.ygcen.2016.05.026

Ruuskanen, S., Hsu, B.Y., 2018. Maternal Thyroid Hormones: An Unexplored Mechanism Underlying Maternal Effects in an Ecological Framework. Physiological and Biochemical Zoology. https://doi.org/10.1086/697380

Ruuskanen, S., Hsu, B.-Y., Heinonen, A., Darras, V., Vainio, M., Sarraude, T., Rokka, A., 2018. A new method for measuring thyroid hormones using nano-LC-MS/MS. Journal of Chromatography B.

Salmón, P., Nilsson, J.F., Nord, A., Bensch, S., Isaksson, C., 2016. Urban environment shortens telomere length in nestling great tits, Parus major. Biol Lett 12, 20160155. https://doi.org/10.1098/rsbl.2016.0155 
Salmón, P., Nilsson, J.F., Watson, H., Bensch, S., Isaksson, C., 2017. Selective disappearance of great tits with short telomeres in urban areas. Proc Biol Sci 284, 20171349. https://doi.org/10.1098/rspb.2017.1349

Sarraude, T., Hsu, B.-Y., Groothuis, T., Ruuskanen, S., 2020a. Manipulation of prenatal thyroid hormones does not influence growth or physiology in nestling pied flycatchers. Physiological and Biochemical Zoology.

Sarraude, T., Hsu, B.Y., Groothuis, T., Ruuskanen, S., 2020b. Testing the short-and long-term effects of elevated prenatal exposure to different forms of thyroid hormones. Peerj. https://doi.org/10.7717/peerj.10175

Schielzeth, H., 2010. Simple means to improve the interpretability of regression coefficients. Methods in Ecology and Evolution 1, 103-113. https://doi.org/10.1111/j.2041210X.2010.00012.x

Schielzeth, H., Dingemanse, N.J., Nakagawa, S., Westneat, D.F., Allegue, H., Teplitsky, C., Réale, D., Dochtermann, N.A., Garamszegi, L.Z., Araya-Ajoy, Y.G., 2020. Robustness of linear mixedeffects models to violations of distributional assumptions. Methods in Ecology and Evolution 11, 1141-1152. https://doi.org/10.1111/2041-210X.13434

Smith, S.M., Nager, R.G., Costantini, D., 2016. Meta-analysis indicates that oxidative stress is both a constraint on and a cost of growth. Ecol Evol 6, 2833-2842. https://doi.org/10.1002/ece3.2080

Stier, A., Bize, P., Hsu, B.Y., Ruuskanen, S., 2019. Plastic but repeatable: rapid adjustments of mitochondrial function and density during reproduction in a wild bird species. Biology Letters. https://doi.org/10.1098/rsbl.2019.0536

Stier, A., Delestrade, A., Bize, P., Zahn, S., Criscuolo, F., Massemin, S., 2016. Investigating how telomere dynamics, growth and life history covary along an elevation gradient in two passerine species. Journal of Avian Biology 47, 134-140. https://doi.org/10.1111/jav.00714

Stier, A., Hsu, B.Y., Marciau, C., Doligez, B., Gustafsson, L., Bize, P., Ruuskanen, S., 2020. Born to be young? Prenatal thyroid hormones increase early-life telomere length in wild collared flycatchers. Biology Letters. https://doi.org/10.1098/rsbl.2020.0364

Stier, A., Massemin, S., Criscuolo, F., 2014. Chronic mitochondrial uncoupling treatment prevents acute cold-induced oxidative stress in birds. Journal of Comparative Physiology B-Biochemical Systems and Environmental Physiology. https://doi.org/10.1007/s00360-014-0856-6

Stier, A., Massemin, S., Zahn, S., Tissier, M.L., Criscuolo, F., 2015. Starting with a handicap: effects of asynchronous hatching on growth rate, oxidative stress and telomere dynamics in free-living great tits. Oecologia. https://doi.org/10.1007/s00442-015-3429-9

Stier, A., Metcalfe, N.B., Monaghan, P., 2020. Pace and stability of embryonic development affect telomere dynamics: an experimental study in a precocial bird model. Proc Biol Sci 287, 20201378. https://doi.org/10.1098/rspb.2020.1378

Tissier, M.L., Williams, T.D., Criscuolo, F., 2014. Maternal Effects Underlie Ageing Costs of Growth in the Zebra Finch (Taeniopygia guttata). PLoS One 9, e97705. https://doi.org/10.1371/journal.pone.0097705

Verhulst, S., 2020. Improving comparability between qPCR-based telomere studies. Molecular Ecology Resources 20, 11-13. https://doi.org/10.1111/1755-0998.13114

Vrijkotte, T.G.M., Hrudey, E.J., Twickler, M.B., 2017. Early Maternal Thyroid Function During Gestation Is Associated With Fetal Growth, Particularly in Male Newborns. The Journal of Clinical Endocrinology \& Metabolism 102, 1059-1066. https://doi.org/10.1210/jc.2016-3452

Welcker, J., Chastel, O., Gabrielsen, G.W., Guillaumin, J., Kitaysky, A.S., Speakman, J.R., Tremblay, Y., Bech, C., 2013. Thyroid Hormones Correlate with Basal Metabolic Rate but Not Field Metabolic Rate in a Wild Bird Species. Plos One. https://doi.org/10.1371/journal.pone.0056229

Wilbourn, R.V., Moatt, J.P., Froy, H., Walling, C.A., Nussey, D.H., Boonekamp, J.J., 2018. The relationship between telomere length and mortality risk in non-model vertebrate systems: a 
bioRxiv preprint doi: https://doi org/10.1101/2021.12.22.473794; this version posted December $22,2021$. The copyright holder for this preprint (which was not certified by peer review) is the author/funder, who has granted bioRxiv a license to display the preprint in perpetuity. It is made available under aCC-BY-NC-ND 4.0 International license.

meta-analysis. Philosophical Transactions of the Royal Society B-Biological Sciences. https://doi.org/10.1098/rstb.2016.0447 Zhang, C., Yang, X., Zhang, Y., Guo, F., Yang, S., Peeters, R.P., Korevaar, T.I.M., Fan, J., Huang, H.-F., 2019. Association Between Maternal Thyroid Hormones and Birth Weight at Early and Late Pregnancy. The Journal of Clinical Endocrinology \& Metabolism 104, 5853-5863. https://doi.org/10.1210/jc.2019-00390

Add: L 